\title{
RESÍDUOS BIOLÓGICOS EM SERVIÇOS DE DIÁLISE: DISCUSSÃO SOBRE O SEU GERENCIAMENTO
}

\author{
DIALYSIS SERVICES' INFECTED WASTE: A DISCUSSION ABOUT ITS MANAGEMENT
}

RESIDUOS INFECTANTES EN SERVICIOS DE DIÁLISIS: DISCUSIÓN SOBRE SU GERENCIA

\author{
Izilda Simões Vitorino Eliam ${ }^{11}$ \\ Izildinha Pedreira Barros ${ }^{2}$ \\ Karla Evangelina Marques Lopes ${ }^{3}$ \\ Anaclara Ferreira Veiga Tipple ${ }^{4}$ \\ Adenícia Custódia Silva e Souza ${ }^{4}$
}

\begin{abstract}
RESUMO: Os profissionais dos serviços de diálise enfrentam situações de riscos desde a realização dos procedimentos diretos às práticas relacionadas, até o gerenciamento dos diferentes resíduos gerados nestes serviços. O acidente com material perfurocortante representa um dos maiores riscos para trabalhadores da saúde. O objetivo foi caracterizar o gerenciamento dos resíduos biológicos nos serviços de diálise da cidade de Goiânia GO. Estudo descritivo, cujos dados foram coletados em março de 2004, por meio de check list elaborado de acordo com RDC 33/ 03/ANVISA, previamente validado, e observados os aspectos éticos e legais, havendo consentimento dos onze serviços existentes. Evidenciou-se que o descarte de perfurocortantes é feito em caixas específicas em quatro unidades; sete unidades improvisam o recipiente, utilizando frascos de soluções dialíticas. Em oito serviços os dialisadores são descartados em sacos brancos leitosos. A segregação de perfurocortantes é feita próxima à fonte geradora na maioria das unidades (91\%). A maioria não possui lixeira com tampa acionada por pedal para descarte de resíduos infectantes. O transporte interno é feito manualmente em $82 \%$ dos serviços. Concluiu-se que na maioria dos serviços pesquisados, as práticas relacionadas ao gerenciamento de resíduos, não atendem às normas de biossegurança, potencializando o risco biológico coletivo.
\end{abstract}

PALAVRAS CHAVES: Resíduos de Serviços de Saúde; Unidades Hospitalares de Hemodiálise; Ambiente.

ABSTRACT: Professionals of the dialysis services face risky situations since from the accomplishment of the pratical procedures to the management of the different kinds of waste generated in these services. Accidents with needlestick materials represent one of the biggest risks for workers of the health. The objective was to characterize the management of infected waste in the dialysis services in Goiânia - GO. This is a descriptive study, with data collected in 2004, march, by check list elaborated accordancing to RDC 33/ 03/ANVISA, previously validated, with ethical and legal aspects observed and legal allowance of the eleven services. It was clearly shown that the discharging of needlestick materials is done specific by four units of service; seven services improvise the container, using bottles of dialysis solution, in order to deischarge the materials. In eight services milky white bags were used to discharge the dialysers. The segregation of the needlestick material waste does happen next to the generating source in the majority of the units $(91 \%)$. The majority of the units didn't have dustbin for infected waste with covers in motion for pedal. The internal transport is made manually in $82 \%$ of the services. This study concluded that in the majority of the researched services, the management of the waste does not follow the biosecurity rules, increasing the collective biological risk.

KEYWORDS: Medical Waste; Hospital Hemodialysis Units; Enviroment.

RESUMEN: Los profesionales de los servicios de diálisis se deparan con situaciones de riesgos desde la realización de los procedimientos directos hasta las prácticas relacionadas, hasta la gerencia de los diferentes residuos generados en estos servicios. El accidente con material corto-punzante representa uno de los mayores riesgos para los trabajadores de la salud. El objetivo fue caracterizar la gerencia de los residuos infectantes en los servicios de diálisis de la ciudad de Goiânia - GO. Estudio descriptivo, cuyos datos fueron recolectados en marzo de 2004, através de check list elaborado según el RDC 33/03/ANVISA, previamente validado, observándose los aspectos éticos y legales, habiendo consentimiento de los once servicios existentes. Fue evidenciado que el descarte de corto-punzantes es hecho en cajas específicas en cuatro servicios; siete servicios improvisan el recipiente, utilizando frascos de soluciones dialíticas. En ocho servicios los dializadores son descartados en bolsas blancas lechosas. La segregación de corto-punzantes es hecha próxima a la fuente generadora en la mayoría de los servicios (91\%). La mayoría no posee basurero con tapa accionada por pedal para descarte de residuos

\footnotetext{
${ }_{1}^{1}$ Enfermeira. Fiscal da Superintendência de Vigilância Sanitária da Secretaria Estadual de Saúde -Goiás.

2 Enfermeira. Mestranda do Curso de Pós Graduação da Faculdade de Enfermagem da Universidade Federal de Goiás. Fiscal da Superintendência de Vigilância Sanitária da Secretaria Estadual de Saúde - Goiás e da Vigilância Sanitária do Município de Goiânia.

${ }^{3}$ Médica. Fiscal da Superintendência de Vigilância Sanitária da Secretaria Estadual de Saúde - Goiás.

${ }^{4}$ Enfermeira. Doutora em Enfermagem. Professor Adjunto da Faculdade de Enfermagem da Universidade Federal de Goiás. (Responsáveis pela correspondência: Rua 227, Qd 68 s/n (FEN/UFG); Setor Leste Universitário; CEP 74075-500; Goiânia - GO. anaclara@fen.ufg.br / adenicia@fen.ufg.br)
} 
infectantes. El transporte interno es hecho manualmente en un $82 \%$ de los servicios. Se concluye que en la mayoría de los servicios investigados, las prácticas relacionadas a la gerencia de residuos no atienden a las normas de bioseguridad, potenciando el riesgo biológico colectivo.

PALABRAS CLAVES: Residuos de Hospitales; Unidades de Hemodiálisis en Hospital; Ambiente.

\section{INTRODUÇÃO}

"A nossa civilização chega ao limiar do século $X X I$ como a civilização dos resíduos” (FERREIRA, 1995, p.314) que tratados de maneira inadequada, tornaramse um dos maiores problemas da atualidade. Integrado a este universo, temos a questão dos Resíduos de Serviços de Saúde (RSS). Conforme o Ministério da Saúde (MS) (BRASIL, 2003, p. 32) são conceituados como:

"resíduos sólidos dos estabelecimentos prestadores de serviço de saúde em estado sólido, semi-sólido resultantes destas atividades. São também considerados sólidos os líquidos produzidos nestes estabelecimentos, cujas particularidades tornem inviáveis o seu lançamento em rede pública de esgotos ou em corpos d'água, ou exijam para isso, soluções técnica e economicamente inviáveis em face à melhor tecnologia disponível".

A Agência Nacional de Vigilância Sanitária (ANVISA) por meio da Resolução Diretoria Colegiada (RDC) $n^{\circ}$ 33 de 25/02/2003 (BRASIL, 2003, p. 8-11) define os RSS em cinco grupos:

"Grupo A (Potencialmente infectantes): Resíduos com a possível presença de agentes biológicos que, por suas características de maior virulência ou concentração, podem apresentar risco de infecção.

Grupo B (Químicos): Resíduos contendo substâncias químicas que apresentam risco à saúde pública ou ao meio ambiente, independente de suas características de inflamabilidade, corrosividade, reatividade e toxicidade.

Grupo C (Rejeitos radioativos): São considerados rejeitos radioativos quaisquer materiais de atividades humanas que contenham radionuclídeos em quantidades superiores aos limites de isenção especificados na norma CNEN-NE-6.02 (BRASIL, 1985).

Grupo $D$ (Resíduos comuns): São todos os resíduos gerados nos serviços abrangidos pela RDC $n^{\circ} 33 / 03$, que, por suas características, não necessitam de processos diferenciados relacionados ao acondicionamento, identificação e tratamento, devendo ser considerados resíduos sólidos urbanos. Grupo $E$ (Perfurocortantes): são os objetos e instrumentos contendo cantos, bordas, pontos ou protuberâncias rígidas e agudas, capazes de cortar ou perfurar."

Os resíduos perfurocortantes quando não manipulados adequadamente podem levar a acidentes que ocasionem a contaminação dos profissionais por diversas doenças veiculadas pelo sangue, como Síndrome da Imunodeficiência Adquirida, Hepatite B, Hepatite C, entre outras; patologias estas, que além de serem estigmatizantes, podem evoluir para fase crônica, afetando a capacidade produtiva de seus portadores e ocasionando a morte. No Brasil 15\% da população já teve contato com o vírus da hepatite $\mathrm{B}$ e os casos crônicos de HBV e HCV devem corresponder a $1,0 \%$ e $1,5 \%$ da população brasileira, respectivamente (BRASIL, 2003a). "O risco de transmissão de patógenos sangüíneos é estimado em $33,3 \%$ para o vírus da hepatite $B$, em $3,3 \%$ para $o$ vírus da hepatite $C$ e em $0.31 \%$ para o vírus da imunodeficiência humana" (FERNANDES \& BARATA, 2004, html).

Os profissionais de saúde dos serviços de diálise enfrentam situações de riscos geradas por situações que incluem desde os procedimentos diretos com o paciente até o reprocessamento com os dializadores e o manuseio dos resíduos ali produzidos. Os resíduos infectantes, segundo classificação da RDC 33/03/ANVISA, devem ser segregados diretamente na fonte de produção e serem acondicionados em recipientes adequados em conformidade com cada tipo de resíduo.

Dentro dos serviços de diálise, segundo Ministério da Saúde (MS) (BRASIL, 2000a), o risco de transmissão de patógenos podem ocorrer desde acidentes com materiais perfurocortantes, equipamentos contaminados, até a veiculação de vírus por meio das mãos ou luvas contaminadas dos profissionais.

Segundo Couto et al (COUTO, 1999, p.566):

"Nas salas de diálise já se isolou HBV de clamps, tesouras, fechaduras de portas, botões de controle de máquinas e de outras superfícies. Estes locais devem ser sistematicamente descontaminados. O profissional da sala de hemodiálise, ao puncionar um acesso de circulação ou aplicar uma injeção em um paciente de diálise, deve ter certeza de que sua luva não tocou esses objetos, que podem estar contaminados e, desta forma transmitir o vírus da hepatite B".

Os profissionais da saúde têm sido identificados como grupo de risco para adquirir infecções ocupacionais. Um estudo realizado com os trabalhadores de unidades de hemodiálises demonstrou elevada prevalência para infecção pelo vírus da hepatite $B$ (VHB) e baixa para o vírus da hepatite $\mathrm{C}$ (VHC) (LOPES,2002). O ambiente dialítico foi apontado como possível fonte de transmissão ocupacional deste vírus, especialmente para o VHB, enfatizando a necessidade de medidas de controle e prevenção adotadas nestas unidades.

Segundo a portaria 82//MS (BRASIL, 2000) e a RDC No 50 /ANVISA (BRASIL, 2002) o serviço de diálise estruturalmente é composto por: consultório médico; sala de recuperação de pacientes; posto de enfermagem e serviços; sala de utilidades; sala para tratamento hemodialítico (neste estudo, entende-se por sala de tratamento hemodialítico, o ambiente onde 
é realizado este procedimento em pacientes HBSAG negativos e acientes HCV positivos); sala para tratamento de pacientes HBsAg positivos; sala para diálise peritoneal ambulatorial contínua - DPAC; sala para diálise peritoneal intermitente- DPI; sala de reprocessamento de dialisadores de pacientes não contaminados por vírus de hepatite; sala de reprocessamento de dialisadores de pacientes HBsAg positivos; sala de reprocessamento de dialisadores de pacientes contaminados por vírus da hepatite $\mathrm{C}$, sala para o tratamento e reservatório de água tratada para diálise; depósito de material de limpeza; sala para armazenagem de concentrados, medicamentos e material médico hospitalar e sanitários para funcionários (ambos os sexos). Não há sala para reprocessamento de dialisadores de pacientes HIV positivos, pois os mesmos são descartados a cada uso.

A maioria desses ambientes gera resíduos biológicos que se não segregados, acondicionados e armazenados adequadamente colocam em riscos os trabalhadores e os pacientes.

O gerenciamento de resíduos de serviços de saúde envolve ações que visam minimizar riscos ocupacionais, e segundo RIBEIRO FILHO (2000, p. 1157) "a base do processo de tomada de decisão é o conhecimento da problemática dos resíduos, suas características e riscos que eles apresentam". Apesar da polemização em torno do real risco biológico gerado pelos resíduos nos serviços de saúde, a literatura é unânime em afirmar que se deve observar cuidados em relação ao manuseio adequado dos mesmos e em especial a atenção com os perfurocortantes.

Um dos pontos principais no gerenciamento consiste na análise e avaliação de riscos, e isto só será possível se houver uma política decisória, em cada serviço, para implementar medidas preventivas que direcionem ações efetivas, visando alcançar resultados que garantam a eliminação e/ou minimização dos riscos.

Um plano de gerenciamentos contempla fases de procedimentos que incluem: segregação, acondicionamento, identificação, abrigo temporário, transporte, tratamento e destinação final.

Com este trabalho pretende-se ampliar as discussões sobre o gerenciamento dos resíduos biológicos dentro dos serviços de diálise, os potenciais riscos e a adequação à legislação sanitária. Para tanto foi estabelecido o objetivo de identificar e analisar o gerenciamento dos resíduos infectantes e perfurocortantes nos serviços de diálise do município de Goiânia-Goiás.

\section{METODOLOGIA}

Este estudo de caráter descritivo exploratório foi desenvolvido no município de Goiânia, estado de Goiás e abrangeu $100 \%$ dos serviços de diálise o que corresponde aos onze estabelecimentos existentes, sendo dois públicos e nove privados.
Os dados foram coletados em março de 2004 e obtidos por meio de observação estruturada, utilizando-se de um check list elaborado com base na RDC n³3/03/ANVISA e previamente validado por pessoas com conhecimento na área de resíduos de serviços de saúde e de biossegurança. O check list foi preenchido durante uma visita em cada estabelecimento, no momento das sessões de diálise e em cada setor da unidade.

A coleta de dados foi feita após a apreciação do estudo por um Comitê de Ética em Pesquisa e consentimento dos diretores da cada serviço de diálise mediante assinatura do termo de consentimento livre e esclarecido conforme Resolução 196/96 do Conselho Nacional de Saúde (BRASIL, 1996).

Os dados foram analisados no programa EPI-INFO 2002 e para discussão foram agrupados nas unidades temáticas:

- Acondicionamento dos resíduos potencialmente infectantes e perfurocortantes.

- Transporte interno dos resíduos.

- Estrutura física dos abrigos para disposição final dos resíduos.

\section{RESULTADOS E DISCUSSÃO}

A etapa mais importante do gerenciamento dos RSS é a segregação que deve ser feita na fonte de geração do resíduo. Esta etapa realizada de forma efetiva permite a minimização de riscos à saúde dos trabalhadores, ao paciente e ao meio ambiente, como também garante a redução dos resíduos infectantes (COUTO, 1999; RIBEIRO FILHO, 2000; BRASIL, 2003).

A RDC 33/03/ANVISA (BRASIL, 2003) preconiza que o resíduo infectante seja acondicionado em saco branco leitoso resistente a ruptura e vazamento, impermeável, segundo a NBR 9191 da Associação Brasileira de Normas Técnicas (ABNT). A mesma resolução preconiza que os perfurocortantes devem ser devidamente separados no local de geração, imediatamente após o uso, em recipiente de paredes rígidas, resistentes a punctura, ruptura e vazamento com tampa baseado na NBR 13853/97 e NBR 9259/97, ambas da ABNT. Em todos os serviços estudados observou-se que estes resíduos são descartados nos locais onde são realizados procedimentos geradores dos mesmos. Os recipientes para o descarte de perfurocortantes encontravam-se geralmente no posto de enfermagem, sobre a bancada de trabalho. Ressalta-se que nos serviços de diálise o tratamento hemodialítico é realizado em ambientes incluindo área para o procedimento técnico com o paciente e área do posto de enfermagem.

\section{1 - Acondicionamento dos resíduos perfurocortantes e potencialmente infectantes:}

O descarte dos perfurocortantes é feito próximo à fonte de produção em dez serviços (92\%). Conforme apresentado na Figura 1, em sete unidades (64\%) o 
Figura - 1 Tipo de recipiente utilizado para o descarte de perfurocortantes dos serviços de diálise, Goiânia, 2004

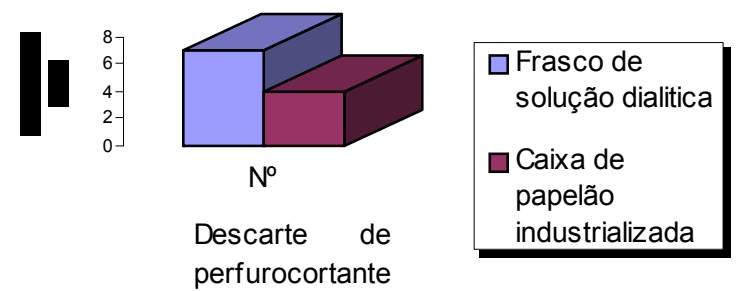

As inadequações do recipiente, que podem ser desde o material dos mesmos até o diâmetro da abertura de acesso ao descarte, colocam em risco a integridade física dos trabalhadores. No caso dos frascos de soluções dialíticas as aberturas não oferecem facilidade para o descarte de perfurocortantes, o que favorece o extravasamento de sangue para a parte externa dos mesmos, fato verificado na maioria das unidades. Observou-se durante a coleta de dados que em nenhuma unidade os recipientes estavam identificados com o símbolo de identificação de resíduos perfurocortantes. $O$ acondicionamento adequado tem por função isolar os resíduos do meio externo, impedir contaminação de resíduos comuns, manter vetores afastados e evitar contato direto dos trabalhadores com os resíduos (RIBEIRO FILHO, 2000; BRASIL, 2001). RISSO (1993) relata um caso onde um catador teve seu dedo amputado em conseqüência de um ferimento com agulha de uma seringa quando rebuscava os resíduos do aterro a céu aberto em um determinado município do estado de São Paulo.

A RDC n³3/03/ANVISA (BRASIL, 2003, p.10) preconiza que "gesso, luvas, esparadrapo, algodão, gazes, compressas, e outros similares, que tenham contato ou não com sangue, tecidos ou fluidos orgânicos, com exceção dos enquadrados na classificação A5 e A7" são considerados como resíduos comuns. Os classificados em A5 são resíduos provenientes de pacientes com contaminação ou suspeitos de conterem agentes contidos em uma tabela (anexa à referida legislação) de vários microrganismos como, vírus da febre aftosa, vírus ebola, etc. A classificação A7 se refere à contaminação por proteína priônica. $\mathrm{Na}$ prática sabemos que não é possível a detecção destes microrganismos em doenças de imediato a não ser por meio de exames específicos. Este é um dos pontos polêmicos da RDC 33/ANVISA/2003, o que está gerando discussões entre os estudiosos do assunto e nos diversos segmentos da sociedade, além do que a Resolução n5/CONAMA (BRASIL, 1993) atualizada pela Resolução $n^{\circ}$ 283/01/CONAMA define esses resíduos como resíduos que apresentam risco potencial à saúde pública e ao meio ambiente devido à presença de agentes biológicos. Nesse caso a segregação desses resíduos junto com os considerados resíduos comuns estaria em conformidade com a legislação, o que correspondem aos onze $(100 \%)$ serviços estudados. A distribuição de lixeiras com tampas acionadas a pedal não é feita de forma homogênea em todos os setores dos estabelecimentos como se pode verificar no tabela 1.

Tabela - 1- Distribuição de presença de lixeira com tampa acionada por pedal nos diversos ambientes do serviço de diálise. Goiânia, 2004.

\begin{tabular}{|l|l|l|l|l|l|}
\hline ESTRUTURA FíSICA & SIM & \multicolumn{3}{|l|}{ NÃO } \\
\hline & $N^{\circ}$ & $\%$ & $N^{\circ}$ & $\%$ \\
\hline Sala de recuperação & 05 & $45 \%$ & 06 & $55 \%$ \\
\hline Sala para tratamento hemodialítico; & 08 & $73 \%$ & 03 & $27 \%$ \\
\hline Sala para tratamento hemodialítico de pacientes HBsAg positivos; & 04 & $44 \%$ & 05 & $56 \%$ \\
\hline Sala para tratamento hemodialítico de pacientes HBsAg negativos & 06 & $54 \%$ & 05 & $46 \%$ \\
\hline Posto de enfermagem & 08 & $73 \%$ & 03 & $27 \%$ \\
\hline Sala de reprocessamento de dialisadores de pacientes contaminados pelo vírus da & 03 & $37 \%$ & 05 & $63 \%$ \\
\hline hepatite C & 03 & $33 \%$ & 06 & $67 \%$ \\
\hline Sala de reprocessamento de dialisadores de pacientes HBsAg positivos & 03 & $75 \%$ & 01 & $25 \%$ \\
\hline Sala de Diálise Peritoneal Intermitente & 01 & $25 \%$ & 03 & $75 \%$ \\
\hline Sala para Diálise Peritoneal Ambulatorial Contínua & &
\end{tabular}


A importância da lixeira com tampa acionada a pedal é evitar o contato manual dos trabalhadores com a tampa, descartar corretamente o resíduo e evitar contato de animais com estes resíduos dentro da unidade. Neste sentido RIBEIRO FILHO (2002, p.1167) chama atenção para que as lixeiras que não forem de uso de áreas administrativas devem possuir "tampa que as fechem hermeticamente para evitar a atração de insetos, o que se dá por meio do cheiro do lixo". Observou-se que em três unidades as salas para tratamento hemodialítico não possuem lixeira com tampa acionada a pedal. A mesma autora coloca que as lixeiras devem ser forradas com sacos plásticos que devem sem padronizados conforme o resíduo que se acomoda em seu interior. Cuidado importante a ser observado é que o volume do lixo não ultrapasse $2 / 3$ da capacidade dos sacos. Observou-se acúmulo de resíduos nas lixeiras em muitos setores dos estabelecimentos que estavam acima da capacidade destas, o que pode dificultar o manuseio dos sacos, nesse sentido a NBR 12809 (ABNT, 1993a), recomenda atenção especial ao se fechar o saco para que o trabalhador não inale o ar produzido em seu interior.

Ressalta-se que nem todas unidades possuem os seguintes tratamentos: pacientes HCV negativos;
HBSAg positivos, Diálise Peritoneal Intermitente, Diálise Peritoneal Ambulatorial Contínua, portanto as unidades que executam estes procedimentos estão distribuídas conforme a Tabela 1. O tratamento hemodialítico dispensado aos pacientes $\mathrm{HCV}$ positivo pode ser feito junto com os pacientes HBSAg negativos, no entanto o reprocessamento deve ter ambientes diferenciados. Como a segregação deve ser feita na fonte de produção estes ambientes devem possuir lixeiras com tampa acionada a pedal para segregação adequada na fonte de produção.

\section{2 - Transporte interno dos resíduos:}

A norma da ABNT NBR 12809 (ABNT, 1993a) preconiza que o transporte de resíduos, cujos recipientes não excedam o volume de 20 litros não necessariamente deverá ser realizado em carrinho, podendo ser feito manualmente, desde que acondicionados adequadamente. Em duas unidades, (18\%) apresentadas na Figura 2, o transporte é realizado em carrinho, por estarem inseridas dentro de hospitais e cujo abrigo final está localizado distante da fonte geradora. Nove unidades $(82 \%)$ realizam o transporte do resíduo, manualmente até o abrigo para acondicionamento final.

Figura 2 - Distribuição do transporte interno do lixo dentro dos serviços de diálise. Goiânia, 2004.

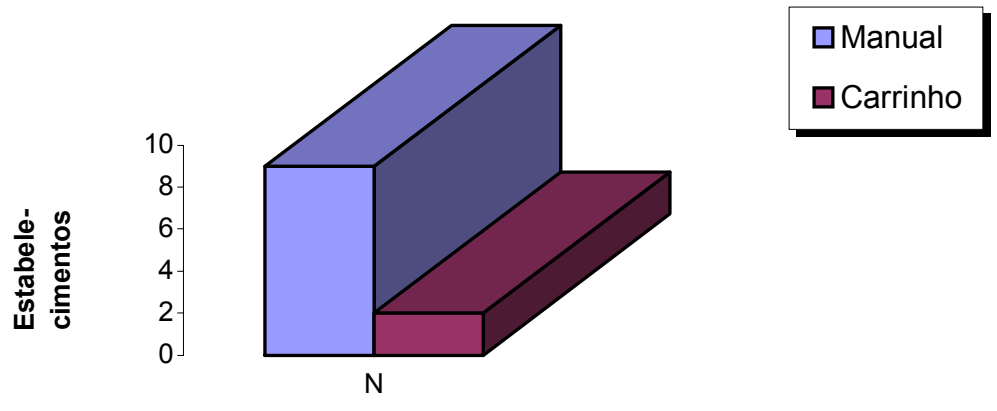

Tipo de transporte utilizado

Os carrinhos destinados ao transporte interno dos resíduos devem ser de uso exclusivo para esta finalidade, mantidos fechados, íntegros e de material que permita a sua higienização. Cuidados devem ser observados para não haver extravasamento vazamento de líquidos, e não oferecer riscos para o trabalhador. É importante que a unidade estabeleça horários definidos para o transporte desses resíduos para não haver cruzamento com visitas, alimentos, roupas limpas e medicamentos (ABNT, 1993).

A RDC 33/ANVISA (BRASIL, 2003, p. 18) preconiza que, quando "o volume de resíduos e a distância entre o local de geração e o armazenamento final justifiquem, o armazenamento temporário poderá ser dispensado". Dez unidades (91\%) não possuem o abrigo temporário, existindo apenas em uma unidade $(9 \%)$. Observou-se que em algumas das unidades onde não existe $\mathrm{o}$ abrigo temporário, o corredor do estabelecimento era utilizado para esta finalidade onde os sacos eram dispostos diretamente no chão. Prática contra indicada porque pode atrair insetos e roedores, além de representar risco para os trabalhadores.

\section{3 - Estrutura física dos abrigos para disposição final dos resíduos.}

O armazenamento externo dos diversos grupos de resíduos deve ser realizado em locais específicos para esse fim, que tenham localização de fácil acesso tanto para os trabalhadores do estabelecimento quanto para os coletores (coleta final dos resíduos), não permitindo o acesso de pessoas estranhas nesses locais, para evitar manipulação por pessoas inabilitadas (BRASIL, 2001a). Todos os abrigos devem possuir símbolos de identificação, com dimensões compatíveis à geração de três dias (RIBEIRO FILHO, 2000). 
Foi observado que apenas uma unidade (9\%) não possui o abrigo final, sendo os resíduos depositados em contêiner. RIBEIRO FILHO (2000) chama atenção para o fato de que os contêineres não devem substituir os abrigos, mas permanecer dentro dos mesmos.
Conforme a tabela 3 , nos dez serviços que possuem abrigo a estrutura física não atendem aos requisitos da RDC 33/03/ANVISA.

Tabela 2 - Estrutura física de abrigos para disposição final de resíduos em serviços de diálise. Goiânia, 2004.

\begin{tabular}{|l|l|l|l|l|}
\hline & SIM & \multicolumn{3}{l|}{ NÃO } \\
\hline & $N^{\circ}$ & $\%$ & $N^{\circ}$ & $\%$ \\
\hline Acesso Interno & 08 & 80 & 02 & 20 \\
\hline Acesso externo & 10 & 100 & - & - \\
\hline Ponto de água & 08 & 80 & 02 & 20 \\
\hline Ralo provido de tampa & 03 & 30 & 07 & 70 \\
\hline Ralo sifonado e ligado à rede de esgoto & 10 & 100 & - & - \\
\hline Piso lavável & 09 & 90 & 01 & 10 \\
\hline Piso impermeável & 04 & 40 & 06 & 60 \\
\hline Aberturas teladas e portas vedadas & 05 & 50 & 05 & 50 \\
\hline
\end{tabular}

O acesso interno ao abrigo é importante para que os funcionários não transitem pela via pública durante a disposição final dos resíduos. Em dois serviços (20 \%) esse transporte é feito em desacordo com a legislação sanitária, uma vez que é feito pelo lado externo do estabelecimento. Todos os abrigos possuem acesso externo para coleta urbana dos resíduos.

Em cinco serviços $(50 \%)$ as aberturas e portas não são teladas. A necessidade de ter todas aberturas teladas, incluindo vedação inferior nas portas é impedir o acesso de insetos e roedores. Em oito (80 \%) serviços não existe ralo com tampa o que facilita, também, a entrada de insetos. Observou-se que em dois serviços $(20 \%)$ não existe ponto de água, em um $(10 \%)$ o piso não é lavável e em seis $(60 \%)$ o piso não é impermeável dificultando a higienização do ambiente, entretanto todos possuem ralos sifonados ligados a rede de esgoto. Ressalta-se que a limpeza adequada do abrigo é fator importante na prevenção de doenças ocupacionais. $O$ acúmulo de resíduos provoca cheiro forte, pode provocar aerossolização de microorganismos, e ser atrativo para animais.

\section{CONCLUSÃO:}

Evidenciou-se que a normativa (RDC33/03/ANVISA), não é cumprida em sua totalidade na realização do gerenciamento dos resíduos nos serviços de diálise de Goiânia.

Diante disso conclui-se que para o cumprimento da legislação é necessário:

- Determinação dos gerenciadores dos estabelecimentos;

- Implantação do Plano de Gerenciamento dos Resíduos de Serviços de Saúde em todos os serviços:

- Treinamento de todos os profissionais que diretamente estão envolvidos com as etapas de coleta, transporte, tratamento, armazenamento dos resíduos e da higienização dos ambientes;
- Adequação dos abrigos, em relação à estrutura física e proteção contra insetos e roedores;

- Adequação das lixeiras para acondicionamento dos resíduos;

- Supervisão constante sobre o manuseio dos mesmos;

- Educação continuada para os profissionais;

- Avaliação de riscos gerados por segregação inadequada.

As principais vantagens da segregação é minimizar riscos de contaminação do meio ambiente, dos trabalhadores, desde a unidade até a coleta final, e evitar o contato dos resíduos comuns com o resíduo infectante, considerando que os resíduos comuns podem ser reciclados (RIBEIRO FILHO, 2000, p..1162).

\section{REFERENCIAS BIBLIOGRÁFICAS}

ABNT - Associação Brasileira de Normas Técnicas. NBR12807. Citações em documentos: apresentação.resíduos de serviços de saúde; ABNT, Rio de Janeiro, 1993.

ABNT - Associação Brasileira de Normas Técnicas. NBR12809. Citações em documentos: apresentação.resíduos de serviços de saúde; ABNT, Rio de Janeiro, 1993a.

BRASIL. Conselho Nacional de Energia Nuclear. Resolução NE-6.0 de 1985. Dispõe sobre Gerência de rejeitos radioativos em instalações radiativas. Diário Oficial da República Federativa do Brasil, Brasília, 17 dez. 1985.

BRASIL, Conselho Nacional de Meio Ambiente. Resolução $n^{\circ} 05$ de 05/08/1993. Define os procedimentos mínimos para o gerenciamento de resíduos sólidos oriundos de serviços de saúde, portos e aeroportos. Diário Oficial da União de 31 de ago. de 1993, Brasília, 1993. 4p. 
BRASIL. Ministério da Saúde. Conselho Nacional de Saúde. Resolução 196/96. Dispõe sobre Diretrizes e Normas Regulamentadoras de Pesquisa Envolvendo Seres Humanos, Brasília, 1996. 09p.

BRASIL, Ministério da Saúde. Portaria $n^{\circ} 82$, de 03/01/2000. Estabelece o Regulamento Técnico para o funcionamento dos serviços de diálise. 2000.

BRASIL. Ministério da Saúde. Agência Nacional de Vigilância Sanitária - Curso Básico de Controle de Infecção Hospitalar - Caderno C - Métodos de Proteção Anti-Infecciosa, Brasília, 2000a.

BRASIL, Ministério da Saúde. Gerenciamento de Serviços de Saúde. Projeto Reforço à Reorganização do Sistema Único de Saúde. Brasília, 2001.

BRASIL, Conselho Nacional de Meio Ambiente. Resolução $n^{\circ} 283$ 12/07/2001. Dispõe sobre o tratamento e destinação final dos resíduos de serviços de saúde. Brasília, 2001a.

BRASIL, Ministério da Saúde. Agência Nacional de Vigilância Sanitária Resolução - RDC $n^{\circ} 50$, 21/02/2002. IANVISA Atualizada pela Resolução RDC n. ${ }^{\circ} 307$, de 14/11/2002 (I) Dispõe sobre o Regulamento Técnico para planejamento, programação, elaboração e avaliação de projetos físicos de estabelecimentos assistenciais de saúde. Brasília, DF, 2002.

BRASIL, Ministério da Saúde. Agência Nacional de Vigilância Sanitária. Resolução RDC $n^{\circ} 33$ 25/02/2003. Dispõe sobre o Regulamento Técnico para o gerenciamento de serviços de saúde. Diário Oficial da União de 05 de mar. de 2003, Brasília. 2003.

BRASIL, Ministério da Saúde. Secretaria executiva. Programa Nacional de Hepatites Virais. Hepatites: O Brasil está atento. Brasília, 2003a.

COUTO, R.M. Infecção Hospitalar. Epidemiologia e Controle. $2^{\mathrm{a}}$ ed. Minas Gerais: MEDSI, 1999. p. 218246.

FERREIRA, J. A. Solid waste and Nosocomial waste: $\mathrm{Na}$ Ethical Discussion. Cad. Saúde Pública, Rio de janeiro, v. 11, n. 2, p. 314-320,1995.

FERNANDES, Antonio Tadeu; BARATA Luiz Carlos Barados. Medicina Baseada em evidências e controle da Infeç̧ão Hospitalar. Princípios básicos para a prevenção das infecções hospitalares. Intervenção 4: Princípios básicos para o uso seguro e descarte adequado de materiais perfurocortantes. Disponível em http://www.ccih.med.brl. Acesso em 29/04/2004.

LOPES, C.L.R. Análise soroepidemiológica da infecção pelo vírus da Hepatite $B$ e $C$ em profissionais dos centros de hemodiálise de Goiânia-Goiás. Resumo de dissertação (mestrado) Revista Eletrônica de Enfermagem (on-line) V4,n1, p.62.2002. Disponível em http://www.fen.ufg.br/revista/revista4 1/hepabc.html Acesso em 29/04/2004.
RIBEIRO FILHO, V. O. Gerenciamento de resíduos de Serviços de Saúde. In: FERNANDES, A. T. et al. Infecção Hospitalar e Suas Interfaces na área da Saúde 2. São Paulo: Atheneu, 2000, 2v. Cap. 63, p. 1157.

RISSO, Wanda Maria de. Gerenciamento de Resíduos de Serviços de Saúde: A caracterização como instrumento básico para abordagem do problema. 1993. 174p. Dissertação (Mestrado). Faculdade de Saúde Pública da Universidade de São Paulo. São Paulo, 1993.

Texto recebido em 24/09/2004

.Publicação aprovada em 10/12/2004. 